LBL- -27581

DE90 001422

\title{
A Method for Measuring the Time Structure of Synchrotron X-Ray Beams
}

\author{
W.W. Moses and S.E. Derenzo, \\ Research Medicine and Radiation Biophysics Division, \\ Lawrence Berkeley Laboratory, 1 Cyclotron Road, \\ Berkeley, California 94720
}

August 6, 1989

\begin{abstract}
Abotrect
We deacribe a method employing a platic scintillator coupled to a fast photomultiplier tube to generate a timing pulse from the $x$-ray bursts emitted from a synchrotron rediation source. This technique is unful for performing synchrotron experiments where detailed knowledge of the timing diatribution is necueary, wuch a time rewolved spectroncopy or fluorencence lifetime experiments. By digitizing the time differente between the timing signal guerred on one bean crowing with the timing sional gererated on the next beam crosing the time atructure of a synchrotron beam can banalyzed. Using this technigus, we have invetigated the single bunch time structur at the National Synchrotron Light Source (NSLS) during pilot rung in Jenuary, 1989, and found that the majority of the beam (90\%) is contained in one If bucket, while the reanainder of the beam (4\%) is contained in satellite ff buckets preceeding and following the main if bucket by $19 \mathrm{~m}$.
\end{abstract}

In order to perform timing experiments such en time realved spectroncopy or fluorescence lifetime measurements at synchrotron radiation facilitien, it is neceasary to obtain a timing signal that is sccurately synchronised to the incident beam. The most common method for obtaining this timing signal is to sample the radia-frequency (If) signal that powers the synchrotron's accelerating cavitien with a sero-croming discriminator. As the If cavitiea and amociated power applien are unully located many meters away from the experimental asea, a loos cable is required to carry the timing sigmal to the experimental area, frequently resulting in electrical noive and subsequent timing jitter because of ground loops and antenna effects. In addition, the time difference between the if crossover and 
the arrival of the $x$-ray beam into the experimental area is unknown, and can vary during a run as the accelerator orbits are changed.

Therefore, we have devised a method the uses the $x$-ray beam itself to generate a timing pulse. The device, shown in Fig. 1 consists of a thin (0.043 in. thick $\times 0.375$ in. wide $\times$ 1.0 in. long) piece of Pilot U plastic scintillator coupled with Dow Corning 3140 RTV to a fast (2.3 na rise time, $1.6 \mathrm{~ns}$ transit time spread) Hamamatsu R647-01 photomultiplier tube that is powered by a Hamamatsu E849-02 base. The platic scintillator is wrapped with - reflective conting of aluminum foil, and the entire amembly is wrapped with black tape to block ambient light. When the scintillator is placed in the path of the $x$-ray beam, the $x$-rays excite the scintillator and cave the photomultiplier tube to fire. An Ortec 473A coastant fraction discriminator converts the photomultiplier tube output into a timing signal that is accurately synchronized to the leading edge of the synchrotron x-ray beam.

The intrinic timing resolution of this device is meanured by constructing two such devices, supplying $-1100 \mathrm{~V}$ to each of their beses, and placing a positron emitting ${ }^{*} \mathrm{Ge}$ source between them. The back-to-back pair of 511-keV photons reaulting from positron annihilation excites the two devices aimultaneously, and the difference between the arrival times of the two pulses is meanured with an Ortec 457 time to amplitude convmiter and digitized with a LeCroy 3512 analog to digital converter. The reoulting distribution, shown in Fig. 2, has a full width at half maximum (FWHM) of $1.5 \mathrm{~ns}$, which implies that the time resolution of a single device has a FWHM of $1.2 \mathrm{~ns}$.

This device is usually placed to one side of our sample in a portion of the incident $x$-ray beam that is not being ued to irradiate the sample. In this configuration, it can be uned with $x$-ray energies a low a a few $\mathrm{keV}$, limited by the $x$-ray beam attenuation in the aluminum foil and black tape surrounding the scintillator. A minimum total energy deposit in the scintillator of approximately $100 \mathrm{keV}$ is neceseary to form a good timing pulse. Using this value and the mam abeorption cross section for aluminum [1], Fig. 3 shows the minimum flux necessary to form a good timing signal, in units of number of $x$-rays 
per bunch incident on the det:zctor, as a function of the incident $x$-ray energy. At $5 \mathrm{keV}$ incident energy, an incident flux of approximately 340 photo:ss per bunch is necessary.

As the $\mathrm{x}$-ray energy increases, their penetrating power increases to the pnint where most of the $x$-rays pase through the scintillator without interacting. Fig. 3 also shows the fraction of the incident $x$-ray beam that passes unaltered through the device as a function of energy. Less than $50 \%$ of the incident beam flux of $x$-rays above $10 \mathrm{keV}$ is abeorbed in the scintillator and aluminum coating. Therefore the device can be placed in the $x$-ray beam in front of the eample without aignificantly affecting the beam, and the minimum incident flux is determined not by absorptin." a "lis srotective wropping but by

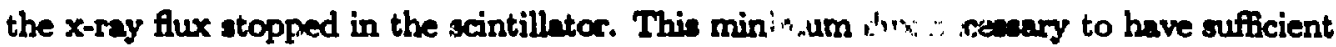
radiation abeorbed by the scintillator is a slowly varying function of the x-ray energy, and is approximately 100 photons per bunch impinging on the device for $x$-ray energies above $6 \mathrm{keV}$.

We used this device to investigate the beam timing structure with monochromatic $22 \mathrm{keV} x$-ray radiation from beamline X23-A2 at the National Synchrotron Light Source (NSLS) during pilot single bunch run in Janiary, 1989. The time difference between timing signals generated by our device for succesive bunch crossings is digitized with the same equipment used to measure the intrinic timing resolution of the device, and the resulting distribution is shown in Fig. 4. The majority of the beam (96\%) is contained in one if bucket, while the remainder of the beam (4\%) is distributed into two satellite If buckets located $19 \mathrm{~ns}$ preceeding and following the main if bucket. When the effect of the intrinsic timing resolution of our device is subtracted from the $2.3 \mathrm{~ns}$ FWHM of the central peak, we measure a $1.9 \mathrm{~m}$ FWHM variation in the bunch rotation period.

In concluaion, we have constructed a simple device based on a photomultiplier tube and plastic scintillator that provides a timing pulee synchronized within $1.2 \mathrm{~ns}$ of the arrival time of an $x$-ray pulse. The device is uneful for $x$-ray energies above a few $k e V$, and can operate with fluxes as low as a few tens to hundreds of $x$-rays impinging on the device. In 
addition to providing a timing pulse for fluorescence lifetime or time resolved spectroscopy measurements, it can be used to measure the synchrotron $x$-ray beam time structure.

We would like to thank John Cahoon of Lawrence Berkeley Laboratory for invaluable technical support, as well as Dr. Chuck Bouldin of the National Bureau of Standards for use of Beam Line X23-A2 at NSLS.

This work was supported in part by the Director, Office of Energy Research, Office of Health and Environmental Research of the U.S. Department of Energy, under contract No. DE-AC03-76SF00098, and in part by Public Health Service Grant Numbers P01 HL23840 and R01 CA38086 awarded by the National Heart, Lung, and Blood Institute and the National Cancer Inatitute of the U.S. Department of Health and Human Services. The research was carried out in part at the National Synchrotron Light Source, Brookhaven National Laboratory, which is supported by the U.S. Department of Energy, Division of Materials Sciences and Division of Chemical Sciences, under contract No. DE-AC02$76 \mathrm{CHOOO16.}$

Reference to a company or product name does not imply approval or recommendation of the product by the University of California or the U.S. Department of Energy to the exclusion of others that may be suitable. 


\section{References}

[1] B.L. Henke, J.C. Davis, E.M. Gullikson, and R.C.C. Perera, A Preliminary Report on $X$-Ray Photoabsorption Coefficients and Atomic Scattering Factors for 92 Elements in the 10-10,000 eV Region, Lawrence Berkeley Laboratory report LBL-26259, (1988). 
LBL-27581

6

List of Figures

Figure 1: Photograph of the plastic scintillator $(0.043$ in. $\times 0.375$ in. $\times 1.0$ in. $)$, photomultiplier tube ( 0.5 in. diameter $\times 2.8 \mathrm{in.} \mathrm{long}$ ), and base (1.375 in. long) before the assembly was wrapped with black tape.

Figure 2: Coincidence time distribution of two devices excited simultaneously.

Figure 3: Minimum flux (number of $x$-rays per bunch incident on the device) necessary to produce a good timing signal and fraction of incident beam transmitted as a function of incident $x$-ray energy.

Figure 4: Beam timing structure measured during pilot single bunch runs at NSLS in January, 1989. 


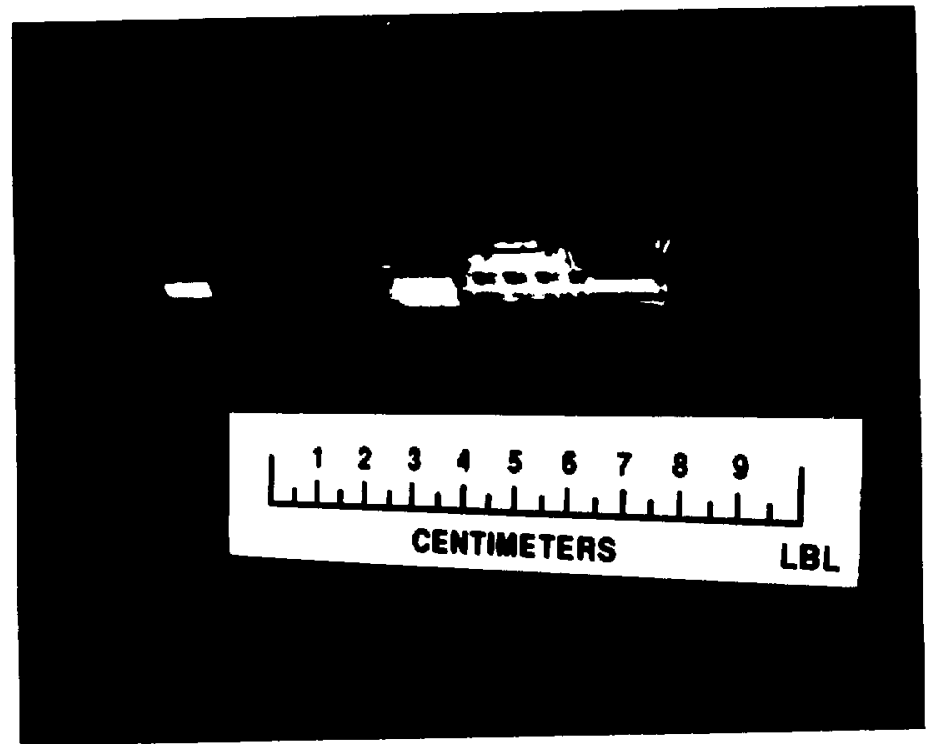

CBB 897-6096

Figure 1 


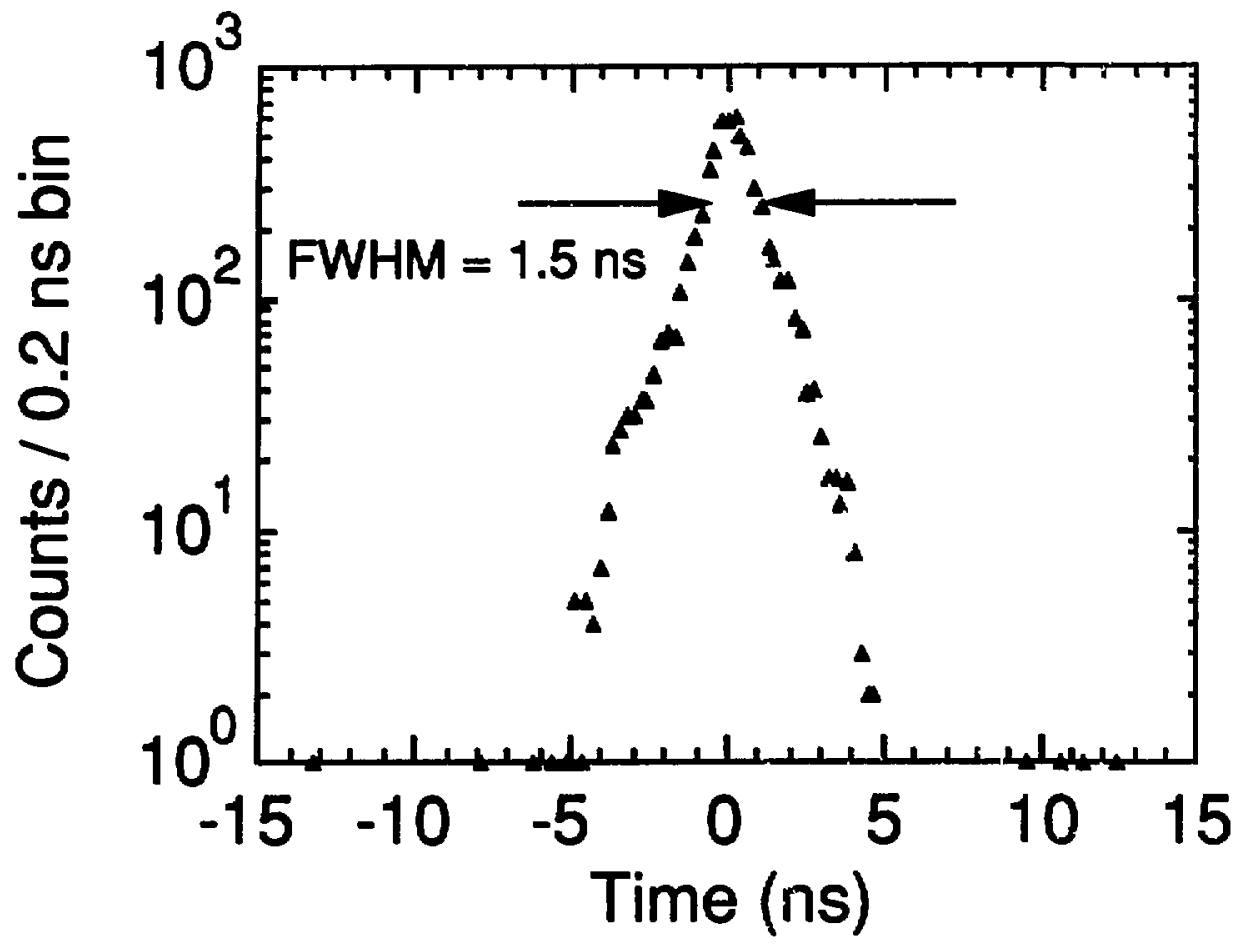

XBI. 898-2952

Figure 2 


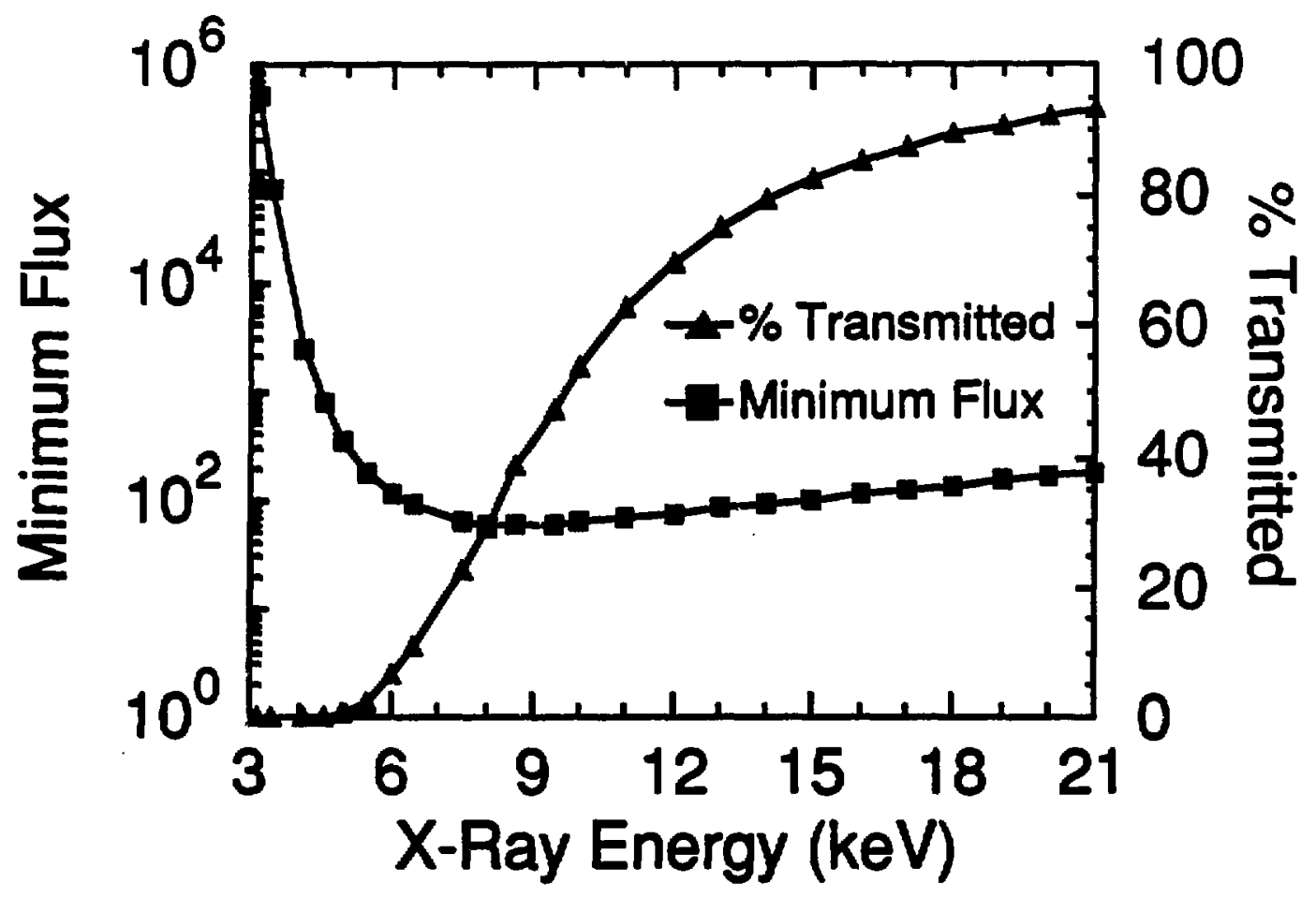

XBL 898-2951

Figure 3 


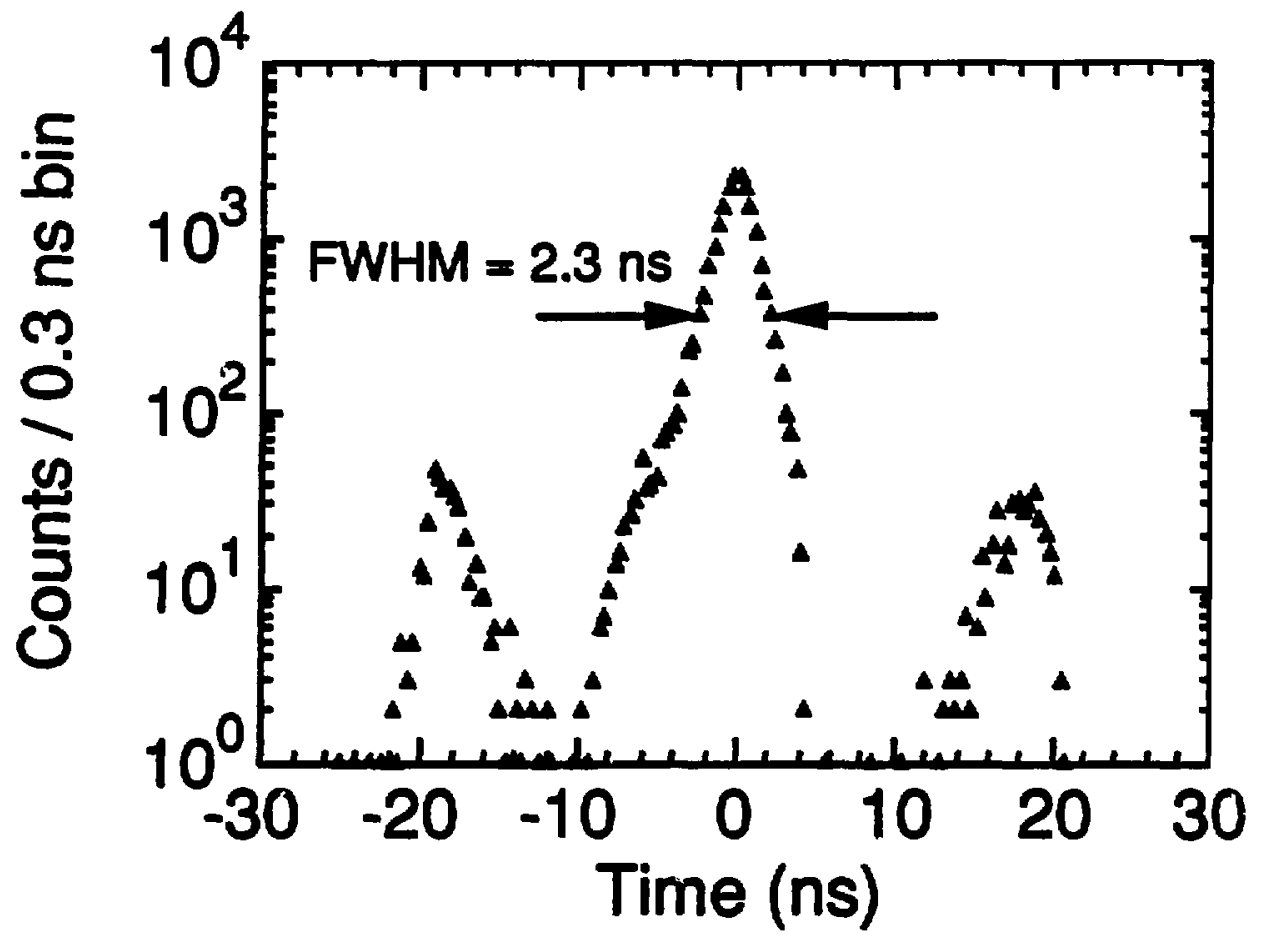

XBL 898-2950

Figure 4 